\title{
XVII.
}

\section{Ueber das Vorkommen der Fissura mastoidea squamosa und deren praktische Bedeutung.}

\author{
Von \\ Dr. Wilh. Kirehner, \\ praks. Axzt in Wuraburg,
}

(Hierzu Tafel III.)

Es ist eine in der Praxis häufig zu beobachtende Erscheinung bei acuten Entzündungsprocessen in der Paukenhöhle, dass die Erkrankung nach aussen hin auf die Gegend des Warzenfortsatzes fortgeleitet wird und hier als Periostitis, Zellgewebsentzündung oder unter den Erscheinungen eines einfachen Abscesses sich abspielt, manchmal jedoch anch die Veranlassung zu weitgehender Zerstörung des Knochens durch Caries und Nekrose liefern kann. Minder häufig dagegen lässt sich bei dem einfachen chronischen Paukenhöhlenkatarrh das Vorkommen von Abscessbildung am Warzenfortsatze constatiren, ferner dürte wohl sehr selten diese Complication im letzteren Falle zn tief greifenden Läsionen des Knochens am Warzenfortsatze führen, wie dies häufiger im Gefolge von acuten und chronisch-eitrigen Mittelohrprocessen zu geschehen pflegt. Diese Complication von Abscessen am Warzenfortsatze mit einfachem chronischem Paukenhöblenkatarrh erregte meine Aufmerksamkeit bei mehreren Fallen, die ich im Jahre 1876 und 1877 zu beobachten Gelegenheit hatte. Es handelte sich dabei um die gewöhnliche, sehr bäufig vorkommende Form des chronischen Paukenhöhlenkatarrhs mit allmählicher Verdickung und Sklerosirung der Schleimhaut in der Pankenböhle. Aus dem höchst einfachen symptomlosen Verlauf der ganzen Erkrankung des Gehörapparates liess sich eine Fortleitung des chronischen Entzündungsprocesses nach aussen gegen die Bedeckung des Warzenfortsatzes hin gar nicht erwarten. Die 
Vorkommen der Fissura mast. squamosa und deren prakt. Bedeutung. 191

Patienten, bei denen diese Complication am Warzenfortsatze auftrat, waren alle im Allgemeinen kräftig und gesund und dem mittleren Lebensalter angehörig; der Paukenhöhlenkatarrh verlief bei allen unter so geringfügigen Erscheinungen von Seite des Gehörorgans und seiner Umgebung, ohne Schmerz, ohne auffallende Zunahme von Schwerhörigkeit, ohne naehweisbare gesteigerte Hyperämie und Secretion im Mittelohr, dass man hier besonders günstige Umstände zur Fortleitung der leichten chronisehen Entziindung der Paukenschleimhaut und der Warzenzellen nach aussen auf die Weichgebilde des Warzenfortsatzes annehmen musste und zwar, wie ich glaubte, durch Vermittelung der manchmal am Warzenfortsatze sich vorfindenden Fissura mastoid. squamosa.

Einer von den erwähnten Fällen betraf einen Oekonomen von 45 Jahren, im Allgemeinen gesund und kräftig; derselbe acquirirte sich bereits vor 6-8 Jahren häufig sehr hartnäckige Nasenrachenkatarrhe, die sich unter abwechselnder Besserung und Verschlechterung mehrere Jahre binschleppten und ihm endlich Beschwerden im linken Ohre verursachten, was ihn veranlasste, mich aufzusuchen. Er klagte hauptsächlich über die bekannten Erscheinungen, Ohrensansen, verminderte Hörfähigkeit und etwas Eingenommenheit des Kopfes. Bei der Untersuchung zeigte sich das Trommelfell trübe, grauröthlich, stark einwärts gezogen. Beim Katheterisiren drang der Luftstrom ziemlich gut olne Rasselgeräusche in die' Trommelhöhle ein. Therapentisch wurde gegen den noch bestehenden mässigen Naso-Pharyngealkatarrh fäglich zweiprocentige Kochsalzlösung mittelst des von v. Tröltsch angegebenen Zerstäubers in den Nasenrachenraum applicirt, ferner wurden allè drei Tage circa 10 Tropfen einer 1/4 procentigen Zinklösung per tubas mittelst des Katheters eingespritzt.

Nach dreiwöchentlicher Behaudlnng besserte sich der Znstand wesentlich, das Gehörvermögen betrug für eine gewöhnliche Taschen-Cylinderuhr $40 \mathrm{Cm}$. gegen $5 \mathrm{Cm}$. bei der ersten Untersuchung. Das lästige Ohrensausen hatte sich zwar erbeblich vermindert, war jedoch noch nicht vollständig beseitigt, die Eust. Röhre war beim Katheterisiren in der gewöhnlichen Weise für den Luftstrom permeabel. Während also die Erkrankung der Paukenhöhle noch nicht vollständig als beseitigt zu betrachten war, nahm Patient seine gewolnte Beschäftigung und Lebensweise als Landmann wieder auf, ohne ron Seite seines Gehörs 
in störender Weise incommodirt zu werden. Allein bereits nach Verlauf von zwei Monaten verschlechterte sich sein Zustand in der Weise, dass die subjectiven Geräusche an Intensität immer mehr zunahmen, während sein Gehör gleich gut sich verhielť.

Er stellte sich daher mir wieder vor, um von seinem lästigen Ohrensausen befreit zu werden. Bei der vorgenommenen Untersuchung zeigte sich das Trommelfell trübe, von grauröthlicher Farbe, ohne bemerkenswerthe stärkere Einziehung, die Eustachi'sche Röhre erwies sich beim Katheterisiren für den Luftstrom in gewöhnlicher Weise durchgängig, die lange Zeit bestandene Schwellung, and Hyperämie im Nasenrachenraume war jetzt vollständig verschwunden. Die Gegend des Processus mast. war bei Druck völlig schmerzlos, im Ohre selbst fühlte Patient auch keinerlei Schmerz, seine Hauptbeschwerde bildete jetzt nur das lästige Ohrensausen. Therapentisch wurde weiter nichts vorgenommen, als täglich einmal die Luftdouche mittelst des Tubenkatheters ausgeführt, was jedesmal ein Gefühl von Erleichterung und Nachlass der subjectiven Geräusche bewirkte, sein Gehör blieb in derselben Weise verhältnissmässig gut wie dies nach der ersten Behandlung der Fall war. Während allmählich die lästigen Geräusche im Ohre nachliessen, bemerkte ich zu meiner grossen Ueberraschung nach Verlauf von 14 Tagen, ohne dass Patient zuvor iber Schmert, Spannung, oder sonst unangenehme Empfindungen im Ohre oder am Warzenfortsatz geklagt hätte - an letzterer Gegend eine diffuse Röthe; die Partie war etwas geschwellt, bei Druck nicht schmerzhaft. 3-4 Tage später nahm die Geschwulst zu, starker Fingerdruck verursachte mässigen Schmerz und man konnte durch das Gefühl Eiteransammlung in der Tiefe constatiren, wie dies in der That durch einen Einschnitt sich erwies. Es entleerte sich eine mässige Menge gutartigen Eiters, die Sonde traf in der Wunde nirgends auf Rauhigkeiten am Knochen, sondern die Theile fiihlten sich in der Tiefe ganz glatt an, eine tiefere Verletzung des Knochens. mit Ablösung oder Zerstörung des Periostes war also hier auszuschliessen. Unter der gewöhnlichen Behandlung mit Carbolausspritzungen and Umschlägen heilte die Wunde in fünf Tagen vollständig, die Partie' war bei Druck völlig schmerzlos, die Röthe in der Umgebung verschwunden.

Nach vier Wochen trat der nämliche Process am Warzenfortsatze auffallender Weise noch einmal in derselben Weise und gleichfalls unter den unbedeutendsten symptomen auf. Das 
Gehörvermögen war zur Zeit dieser Complicationen in derselben Weise wie friher geblieben. Eine Zunahme von Schwellung und Hyperämie in der Paukenhöhlenschleimhaut, wie dies bei acuten Nachschüben öfters beobachtet wird, liess sich in diesem Falle nicht constatiren.

Seit $1^{1 / 2}$ Jahren kam kein Rückfall mehr vor. Die Gehörfähigkeit blieb in derselben Weise constant, ca. $30 \mathrm{Cm}$. für eine gewöhnliche Cylinderuhr und 6 Meter fur laut Gesprochenes. Subjective Geräusche sind sehr selten. Der Process in der Paukenhöhle ist zur Zeit als stationär zu betrachten, das Trommelfell ist getrübt, der Luftstrom streicht beim Katheterisiren mit breitem blasenden Geräusch durch die Eustachi'sche Röhre.

Zwei andere Fälle, die mir wegen der Complication von chronischem Paukenhöhlenkatarrh mit Affection der äusseren Bedeckung des Warzenfortsatzes von Interesse und der genaueren Betrachtung wïrdig erschienen, verliefen im Allgemeinen in derselben Weise und unter ähnlichen, bei obigem Falle erwähnten Symptomen, so dass ich deren ausfubrlichere Beschreibung hier unterlassen kann; nur so viel sei dariber bemerkt, dass in dem einen Falle, bei einer Frau von 40 Jahren, die Abscessbildung auf dem Warzenfortsatze, ohne Beschädigung des Knochens, sich dreimal in vierwöchentlichen Pausen wiederholte und immer zugleich mit dem Beginne der Menses zusammenfiel. Die Schwerhörigkeit hatte bei ihr schon einen hohen Grad erreicht und es liessen sich bei der Besichtigung des Trommelfells, ferner beim Katheterisiren der Eustachi'schen Röhre die für die sogenannte Sklerose der Paukenhöhle charakteristischen Zeichen nachweisen, das Trommelfell bot eine weissgraue, matte Färbung dar, der Luftstrom drang beim Katheterisiren der Eustach'schen Röhre sehr laut und breit in die Paukenhöhle. Das Ohrenleiden soll nach Angabe der Patientin in Folge sehr häufiger, in früheren Jahren überstandener Nasen-Rachenkatarrhe entstanden sein. Während sie jedoch vor 5-6 Jahren noch verhältnissmässig gut hörte und dieser Zustand viele Jahre bereits gleichmässig und ohne Séhwankung bestanden haben soll, nahmen die subjectiven Geräusche und zugleich die Schwerhörigkeit in den letzten 3 bis 4 Jahren sehr rasch zu, so dass bei der ersten Untersuchung eine gewöhnliche Cylinderuhr nur sehr schwach beim Andrücken an das Obr, laute Sprache auf 1 Meter Entfernung schwer gehört werden konnte.

Bei dem anderen Falle, einem Manne von 50 Jahren, verlief 
der Process in derselben Weise, die Abscessbildung am Warzenfortsatz trat nur einmal auf und wiederholte sich später nicht wieder. Es handelte sich hier gleichfalls um jene Form von Pankenhöhlenerkrankung, die man mit dem Namen Otitis hypertrophica sive sclerotica bezeichnet. In den letzten Jahren hatte der Patient noch häufig an subacuten Nachschüben mit zeitweiser Besserung and Verschlechterung des Gehörs gelitten, ferner bestanden $a b$ und zu hartnäckige Nasen-Rachenkatarrhe.

Es ist nun eine auffallende Thatsache, dass sowohl bei den einfachen chronischen Ohrkatarrhen, die jenes traurige Stadium der sogenannten Sklerose der Paukenhöhle noch nicht erreicht haben, als anch bei letzterer Form selbst, die leichten subacuten Nachschübe, welche die Schleimhant der Pankenhöhle und der Eustachi'schen Röhre ab und zu in einen leichten hyperämischen, entziindlichen Zustand versetzen, diesen entzïndlichen Process so leicht nach aussen auf die Gegend des Warzenfortsatzes fortleiten und zwar in der Weise, dass sich hier ganz allmählich, fast symptomenlos oder nur unter sehr geringfïgigen Erscheinungen eine diffuse Röthe und Schwellung ausbreitet, die allmählich wieder verschwinden kann oder anch zu Eiteransammlung unter oder uiber dem Periost führt, während dagegen in Fällen von acuten und chronischen eitrigèn Ohrkatarrhen, wo wir die Schleimhaut des Mittelohres hochgradig entziundet, mit Granulationen oder Polypenbildung bedeckt finden, nach jahrelangem Bestehen profuser Otorrhöen eine Uebertragung der Entzündung auf die äussere Fläche des Warzenfortsatzes in der oben gesehilderten Weise sehr häufig nicht stattfindet.

Zur Erklärung dieser Complication von Erkrankung des Periostes oder des epiperiostalen Bindegewebes am Warzenfortsatze bei chronischen katarrhalischen Processen der Paukenhöhle dürfte die Betrachtung der anatomischen Beschaffenheit dieser Gegend am meisten Aufschluss geben und zwar das Verhalten der Fissura mastoidea squamosa, welehe am Warzenfortsatze jugendlicher und alter Individuen sich nicht selten gut erhalten vorfindet. Am Schläfenbein des Neugeborenen findet sie sich constant als dentlich ausgesprochene Furche an der Verbindungsstelle des Warzentheils mit der Schuppe. Auf die praktische Bedeutung dieser Furche und deren Beziehung zum Warzenforsatze und za Erkrankungen der Paukenhöhle hat zuerst Gruber')

1) Wiener medicin. Wochenschrift. 1867. S. 851 . 
aufmerksam gemacht. Ihre gänzliche oder theilweise Persistenz im ausgebildeten Schläfebeine lässt sich dadurch erklären, dass die beim Fötus noch getrennten Theile des Schläfebeines, Schuppen- und Felsentheil nach der Geburt sich knöchern vereinigen, jedoch zuweilen an einzelnen Stellen, wo die knöcherne Vereinigung nicht zu Stande kam, Löcher und Spalten als Spuren der fruberen Trennung hinterlassen. Betrachtet man diese. Verhältnisse näher am Schläfe beine eines Neugeborenen, so geschieht die Zusammensetzung der einzelnen hier in Betracht kommenden Knochen des Schläfebeines in der Weise, dass das äussere Blatt des Schuppentheiles sich mit seinem hinteren Rand an die dem späteren Warzenfortsatze entsprechenden Gegend anlegt, wodurch die als Fortsetzung der Paukenhöhle zu betrachtende, oben und hinter ibr gelegene Warzenhöhle - Antrum mastoideum - von aussen her gedeckt wird. Die zwischen den beiden sich aneinander legenden Knochenstitcken bestehende Furche - Fissura mastoidea squamosa - fuhrt in das Antrum mastoideum und zieht auf der Mitte des Warzenfortsatzes von oben hinten nach vorn unten bis zum Foramen stylomastoid. herab.

Dass von Seite der Anatomen dieser für die praktische Otiatrik wichtigen Furche und deren Beziehung zum Warzenfortsatze früher keine besondere Aufmerksamkeit geschenkt wurde, scheint hauptsächlich darin begrindet zu sein, dass die Zusammensetzung des Schläfebeines bis in die neuere Zeit von den verschiedenen Anatomen verschieden angegeben wurde, ferner dass als untere Grenze der Pars squamosa die am oberen Rande des Porus acust. extern. nach rückwärts ziehende Knochenleiste (Linea tempor.) angenommen wurde.

Die Eintheilung des Schläfenbeines in Schuppe, Felsentheil and Warzentheil stammt von Albinus; Sömmering und Blumenbach nahmen später die Eintheilung in Schuppe und Felsentheil an. A rnold theilt auf Grund der Entwicklungsgeschichte und der vergleichenden Anatomie das Schläfenbein zuerst in Schuppe, Felsentheil und Paukenbein und weist die anderen Eintheilungen als unriehtig zurïck. In seinem Handbuche der Anatomie, Bd. I. S. 394 findet sich auch eine schöne Abbildung der gut erhaltenen Fissur. mastoid. squam., bei deren Erklärung angegeben ist, dass an diesem Schläfenbein die Zusammensetzung aus drei einzelnen Theilen noch theilweise zu erkennen sei. Auch in älteren anatomischen Werken finden sich schöne Abbildungen dieser Furche am Warzenfortsatze, so in Du Verney's Traité 
de l'organe de l'onie. 1731. Tab. XV. Fig. 2, in Cloquet's Anatomie de l'homme. 1821. Tab. XIV und XXVI.

L. Joseph weist auf diese Furche als Residuum der fruheren Trennung des Schläfenbeines in die drei der Entwicklungsgeschichte entsprechenden Theile hin und gibt an, dass sich fast immer eine, wenn auch unvollkommene Naht zwischen Schläfenund Felsenbein vorfindet, welehe sich mitunter auf Horizontalschnitten mehrere (bis zu 7) Millimeter tief nach vorn und innen verfolgen lässt (Zeitschrift für rationelle Medicin. 1866. S. 103).

A eby bespricht in seinem Lehrbuch der Anatomie (Der Ban d. menschl. Körpers. 1868. S. 206) ausfühlhlich die Entstehung der Furche durch Verbindung des Felsenbeines mit der Schuppe. Zwei Abbildungen zeigen sehr schön die gut erhaltene Fissur, in Figur 87 blieb die Verschmelzing der Schuppe mit dem Felsenbein aus, und der Warzenfortsatz zeigte sich in einen vorderen Schuppen- und hinteren Felsentheil geschieden; im Einklange hiermit fehlte auch die Verbindung der beiderseitigen Zellräume, die sich unabhängig ron einander in den Paukenraum öffneten.

An ausgebildeten Schläfenbeinen Erwachsener, welche die Furche ganz vollständig erhalten zeigen, ist der Warzenfortsatz durch dieselbe gleichsam in zwei ungleiche Hälften getheilt, von denen die hintere die vordere an Breite etwas übertrifft, ferner lässt sich an frischen Schläfebeinen mit noch erhaltenen Weichtheilen ein starker Streifen von Bindegewebe, der mit dem Periost fest zusammenhängt, durch die genannte Fissur hindurch in die Zellen des Warzenfortsatzes verfolgen, wodurch eine Verbindung des Periosts am Warzenfortsatze mit der tieferen Schichte der Schleimhaut in Warzenzellen und Paukenhöhle, wo die Schleimhaut zugleich die Stelle des Periostes vertritt, hergestellt wird. Auf diesem Wege lässt sich demnach durch Vermittelung der Blutbahnen die Thatsache erklären, dass, abgesehen von den tief greifenden, den ganzen Organismus in Mitleidenschaft ziehenden Affectionen der Paukenhöhle und der Warzenzellen, auch leichte entzitindliche Processe dieser Theile obne bemerkenswerthe subjective und objective Erseheinungen auf die äussere Bedeckung des Warzenfortsatzes übertragen werden können. Es isț daher auch dem Warzenfortsatze und den in ihm enthaltenen lufthaltigen Räumen - wie auch Bezold in diesem Archiv Bd. XIII. Heft 1 betont hat - in Bezug auf deren selbständige Erkrankung eine grössere Bedeutung beizulegen, als man bis jetzt gewohnt war. Ein entzïndlicher Process, der ursprünglich von der Pauken- 
höhle ausgehend in die Warzenzellen tibertragen worden war, kann sich an der ersten Stelle in Folge der günstigeren anatomischen Beschaffenheit dieser Theile bereits wieder zurückgebildet haben, während in den mehr abgeschlossenen und schwerer zugänglichen Warzenzellen der nämliche Process noch längere Zeit fortbesteht und sich hier gleichsam als selbständige Localerkrankung abspielt.

Da dieše Erkrankung am Warzenfortsatze und deren Beziehung zu. der erwähnten Fissura mastoidea squamosa von praktischer Wichtigkeit ist, so dürfte es nicht iuberflüssig erscheinen, diese Verhältnisse einer näheren Betrachtung zu unterwerfen. Ich nahm deshalb im hiesigen anatomischen Institute die Untersuchung einer, wenn auch nicht sehr grossen, so doch immerhin brauchbaren Anzahl von Schädeln Erwachsener und Kinder vor, um statistisch festzustellen, in welcher Häufigkeit die genannte Fissur entweder vollständig oder theilweise erhalten sich vorfindet.

Für die freundliche Erlaubniss, das Material der anatomischen Anstalt zu benutzen, spreche ich Herm Geheimrath ron Kölliker meinen verbindlichsten Dank aus, ebenso fühle ich mich Herrn Prosector Dr. Flesch für seine freundliche Unterstützung zu grossem Dank verpflichtet.

Das mir zu Gebote stehende Material bestand aus 300 Schädeln Erwachsener von versehiedenen Altersstufen und 30 Kinderschädeln, meistens Neugeborene, einige im Alter von 4,6 und 8 Jahren.

Betrachten wir zuerst die Schädel Erwachsener, so findet sich folgendes Verhalten:

Die Fissura mastoidea squamosa findet sich bald vollständig erhalten und zwar in der Weise, dass an der Stelle, wo der hintere untere Winkel des 0 s parietale sich mit der Schläfenbeinschuppe vereinigt, gleichsam eine Sutur von dem Aussehen der iibrigen am Schädel vorkommenden Nähte mit glatten Rändern iiber den Warzenfortsatz herab bis zum Foramen stylomastoideum sich herabzieht, bald unvollständig und in Spuren in der Weise, dass ihr Verlauf durch grössere und kleinere Brücken von soliden glatten Knochenflächen unterbrochen ist, wodurch auf dem Process. mastoid. eine Anzahl in der Längsreihe geordneter, grösserer und kleinerer spaltförmiger Vertiefungen entsteht, bald fehlen die genannten Spalten gänzlich, so dass der Warzenfortsatz bei gewöhnlicher Betrachtung auf der Oberfläche eben und glatt sich erweist. Letztere Fälle bezeichne ich in nach- 
folgender Zusammenstellung als der betreffenden Fissur gänzlich ermangelnde. Doch soll damit nicht gesagt sein, dass hier eine Ueberleitung entziundlicher Processe von der Paukenhöhle resp. den Warzenzellen auf die äussere Fläche des Warzenfortsatzes ausserdem nicht stattfinden könne. Der Warzenfortsatz wird, wie v. Tröltseh nachgewiesen hat, von einer Anzahl feiner Gefässverzweigungen durchsetzt, welche den Vas. emissar. Santorin., den Art. mening. med., occipital, auricul. post. und den entsprechenden Venen angehören. Es können daher pathologische Processe auch auf diesem Wege einestheils auf die Meningen, anderntheils auf die äussere Bedeckung des Warzenfortsatzes übergreifen. Auf Grund der hier bestehenden anatomischen Verbältnisse hat auch v. Tröltseh zuerst genauere. Vorschriften und Indicationen zur Ausfuihrung operativer Eingriffe am Warzenfortsatz (Wilde'scher Schnitt, Anbohrung) aufgestellt (siehe v. Tröltsch, Die Anatomie des Ohres in ihrer Anwendung auf die Praxis. 1860. S. 80. Virchow's Archiv. 1861. Bd. 21).

Eine praktische Bedeutung bei Erkrankungen des Mittelohrs ist ferner auch den von Hyrtl, Retzius, Coster u. A. beobachteten Rarefactionen am Proc. mastoid. beizumessen, wodürch die äussere Lamelle desselben bis zur Transparenz oder ganz durchlöchert erscheint.

Unter den 300 Schädeln Erwachsener fand ich $15 \mathrm{mal} \mathrm{die}$ Fiss. mastoịd. squamos. in der oben geschilderten Weise völlständig erhalten, $8 \mathrm{mal}$ war die Spalte linkerseits vollständig ausgeprägt,. rechts dagegen nur in Spuren erhalten, 5 mal fand sich linkerseits vollkommene Spalte, während der rechte Warzenfortsatz eben und glatt war ohne Andeutung ron Spalten, rechts vollkommene, links theilweise Spalte fand sich viermal, rechterseits vollkommene Spalte, links keine Andeutung einer solchen fand sich bei keinem der untersuchten Schädel, theilweise vorhandene Furchen an beiden Warzenfortsätzen waren an 20 Schädeln vorhanden, theilweise Spalte linkerseits, rechts keine zeigten 12 Schädel, rechts theilweise Spalte, links keine Spur fand sich an 6 Schädeln. Nach dem Procentsatze ausgedrückt würde sich aus dieser Zusammenstellung die Häufigkeit des Vorkommens einer Fissura mastoidea squamosa etwa folgendermaassen ergeben:

1. beiderseitig vollkommene Spalte in 5,0 Proc. der Fälle;

2. links vollkommene, rechts theilweise Spalte in 2,6 Proc. der Fälle; 
Vorkommen der Fissura mast. squamosa und deren prakt. Bedeutung. 199

3. links vollkommene Spalte, rechts nichts in 1,6 Proc. der Fälle;

4. rechts vollkommene, links theilweise Spalte in 1,3 Proc. der Fälle;

5. rechts vollkommene Spalte, links nichts in 0,0 Proc. der Fälle;

6. beiderseitig theilweise Spalte in 6,6 Proc. der Falle;

7. links theilweise Spalte, rechts nichts in 4,0 Proc. der Fälle;

8. rechts theilweise Spalte, links nichts in 2,0 Proc. der Fälle.

Aus diesen Zahlen geht hervor, dass wohl in den meisten Füllen das Vorkommen der Fiss. mastoid. squamosa - sei sie vollkommen oder theilweise erhalten - auf beiden Seiten zugleich stattfindet, bei einseitigem Vorkommen scheint das linke Schläfenbein bevorzugt zu sein, am seltensten scheint Fall 5 vorzukommen, - rechts ausgebildete Furche, links keine Spuren davon - bei obiger Zusammenstellung fand sich dies Verhalten an keinem Schädel. In Bezug auf das Geschlecht lässt sich ein häufigeres Vorkommen nach der einen oder anderen Richtung nicht constatiren. Was das Alter betrifft, so zeigt sich hier, dasś die Fissur sowohl im jugendlichen als im Greisenalter ganz und theilweise vorkommt, unter den untersuchten Schädeln war die Spalte noch zu finden im 68., 70. und 83. Lebensjahr, wo Synostose der übrigen Fissuren und Nähte häufige Befunde sind. An den Kinderschädeln, die wegen der geringen Anzahl keinen Anspruch auf statistische Verwerthung machen können, fand ich die Spalte durchschnittlich bis zum Ablaufe des ersten Lebensjahres vollständig erhalten, nach Ablanf des dritten Lebensjahres fand sich in einigen Fällen keine Spur einer Spalte mehr vor, in anderen noch einige Andeutungen.

Prof. Wiedersheim in Freiburg hat einen in dieser Beziehung sehr interessanten Fall beobachtet, den er Herrn Prosector Dr. Flesch dahier zur Verfügung stellte; Letzterem verdanke ich diese Mittheilung. An einem kindlichen Schläfebein wurde nämlich durch diese Fissur eine so tiefe Rinne erzeugt, dass der Warzenfortsatz wie eingeschnürt erschien und den Eindruck machte, als ob hier eine Verdoppelung desselben vorliege. Als interessanten Befund möchte ich ferner noch erwähnen, dass an einigen Schädeln Erwachsener aus dem mittleren Lebensalter, welche die Fiss. mast. squam. in sehr schön ausgeprägter Weise zeigten, die zwischen Hinterhaupt- und Schläfenbein befindliche Sutura occipito-mastoidea vollständig synostotisch verwachsen 
war, so dass hier gleichsam an Stelle dieser Naht jene uiber den Warzenfortsatz laufende Fiss. mastoid. squamos. erhalten blieb. Dieses Verhalten zeigte sich jedoch nicht ïberall als constantes Vorkommniss.

In Bezug auf die Grössendimensionen des Warzenfortsatzes zeigten die untersuchten Schädel folgende Maasse: durchschnittlich beträgt die Dicke desselben nach dem zwanzigsten Lebensjahre $14 \mathrm{Mm}$, die Höhe $15 \mathrm{Mm}$. und die Breite $18 \mathrm{Mm}$. Diese Zahlen wurden in der Weise gewonnen, dass eine Linie, welche von der Aussenfläche des Warzenfortsatzes bis zur Mitte der Incisur. mastoid. senkrecht durch die Basis des Warzenfortsatzes gelegt wird, als Dicke, eine weitere Linie von der Mitte der Incisur. mast. bis zur Spitze des Warzenfortsatzes gezogen als Höhe und eine dritte senkrecht zur Höhenlinie durch die Basis des Proc. mastoid. von vorne nach rückwärts gelegte Linie als Breite angenommen wurden (vgl. Zoja, Ann. univ. 1864. Maggio).

Im höheren Alter ist eine Abweichung von diesen durchschnittlichen Grössen nicht zu constatiren, dagegen ist an weiblichen Schädeln der Warzenfortsatz durchschnittlich um einige Millimeter kleiner als an männlichen. Die Grössen, welche Zoja bei seinen Untersuchungen an 68 frischen und 100 macerirten Schädeln für den Warzenfortsatz gefunden hat $-13 \mathrm{Mm}$. dick, $12 \mathrm{Mm}$. hoch und $19 \mathrm{Mm}$. breit - differiren von den Zahlen nach meinen Messungen nur um wenige Millimeter.

An Schädeln mit grösseren Spuren oder deutlicher Ausbildung der Fiss. mastoid. squam. zeigte sich der Dickendurchmesser des Warzenfortsatzes kleiner im Verbältniss zum Höhenund Breitendurchmesser; die Dicke desselben betrug in solchen Fällen gewöhnlich nur 10-12 Mm., die Höhe und Breite dagegen 16 und $20 \mathrm{Mm}$.

Nach diesen kurzen Mittheilungen uber das Vorkommen der Fissur. mastoid. squam., deren Beziehung und Bedeutung für Erkrankungen der Paukenhöhle, ist noch der ausgezeichneten Arbeit von Schwartze und Ey sell Erwähnung zu thun, welche den Warzenfortsatz selbst zum Gegenstand eingehender Studien machten (Arch. f. O. N. F. Bd. I). Es wurde zuerst von diesen Autoren die Entwicklung und systematische anatomische Anordnung der im Warzenfortsatze enthaltenen zelligen Räume nachgewiesen, ferner wurde in praktischer Beziehung die hervorragende Bedeutung dieser Theile bei entzündlichen Processen eingehend erörtert, sowie auf die Indicationen und Methoden zu 
erfolgreichen therapeutischen Eingriffen mittelst Eröffnung des Warzenfortsatzes hingewiesen.

Bei dem praktischen Interesse, welches daher der Warzenfortsatz bei entzuindlichen Processen in der Paukenhöhle beansprucht, schien es mir nicht überflüssig, anch diese nicht selten vorkommende Fiss. mastoid. squamosa, die mit den zelligen Räumen des Warzenfortsatzes in Verbindung steht, einer näheren Betrachtung zu unterwerfen, da ohne Zweifel verschiedenartige Erkrankungen dieser Theile des Gehörapparates in ihrem Verlaufe and in ihrer Entwicklung dadurch beeinflusst werden. So z. B. lässt sich der manchmal bei eitrigen Paukenhöhlenkatarrhen angewöhnlich rasch erfolgende Durchbruch des Warzenfortsatzes mit Zerstörung des Periostes und Eiteransammlung unter der äus̄seren Bedeckung des Proc. mast. ganz gut durch theilweises oder vollständiges Bestehen einer Fiss. mastoid. squam. erklären, ebenso wird unsere Aufmerksamkeit auf diesen Zusammenhang gelenkt bei derartigen Vorkommnissen, wie oben erwähnte chronisch-katarrhalische Processe der Paukenhöhle mit Abscessen oder Periostitis am Warzenfortsatze. Beim kindlichen Alter, welches häufiger acuten Erkrankungen der Paukenhöhle ausgesetzt ist, wird wohl diese Furche noch eine grössere Rolle in dieser Beziehung spielen als bei Erwachsenen.

\section{Erklärung der Abbildungen. Tafel 1V.}

Fig. 1-4 F. msq. die Fissura mastoidea squamosa in Fig. 3 nur theilweise, bei den übrigen Abbildungen als vollständige Furche erhalten.

Fig. 3 u. 4 som. Die Sutura occipito-mastoidea in Fig. 1 u. 2 fehlt dieselbe vollständig resp. ist völlig synostotisch verwachsen. 


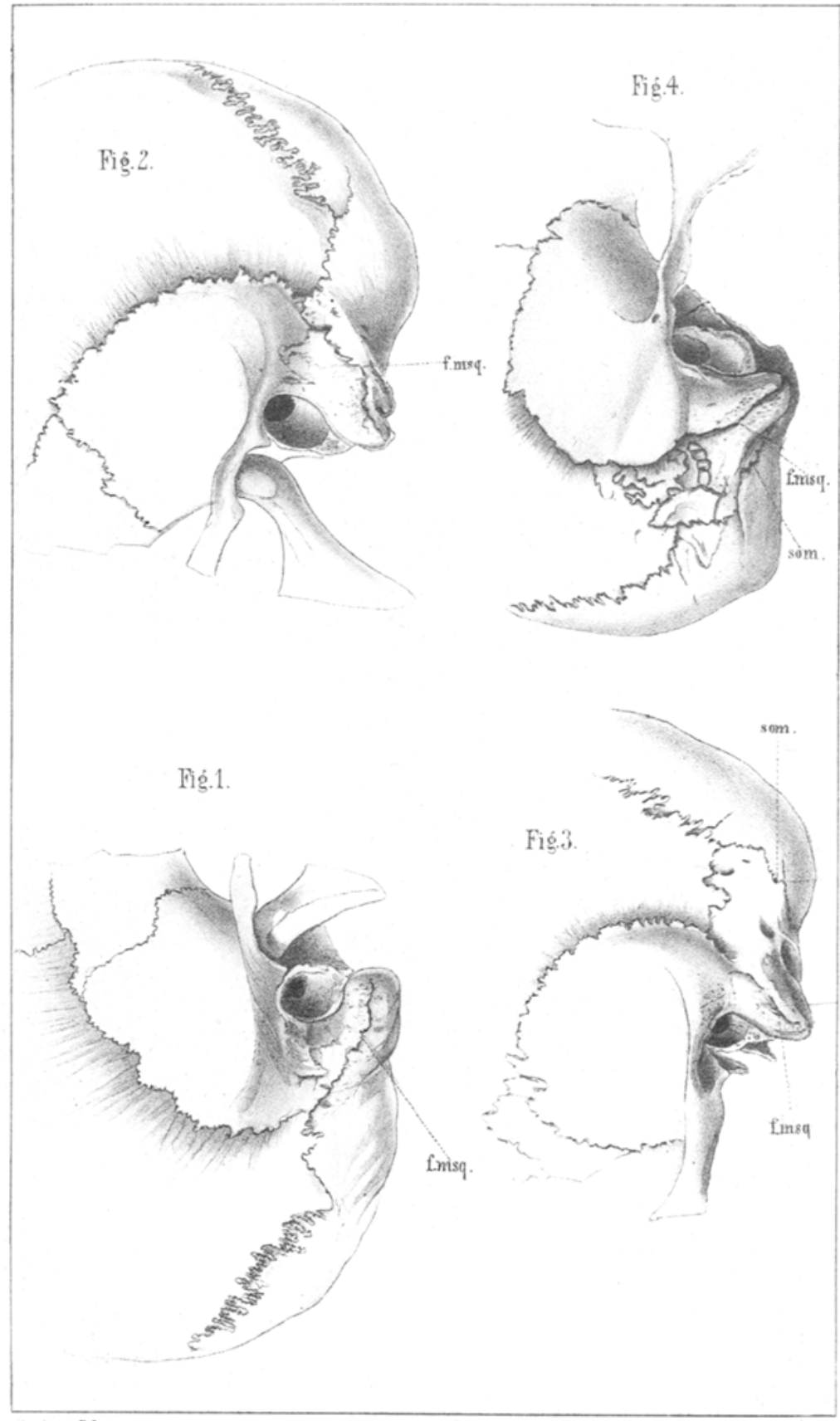

Kirohner,Fifsura mast, squam.

Verlag XFCW.Vogel in Leipsitg.

LithAnst w. GBash,Leipzig 